\title{
Hereditary hemorrhagic telangiectasia with respiratory symptoms at the first stage: two cases report and literature review
}

\author{
yan huang ${ }^{1}$, Rong Huang ${ }^{1}$, bo dou ${ }^{1}$, jian kuang ${ }^{2}$, and chentao liu ${ }^{2}$ \\ ${ }^{1}$ Xiangya Hospital Central South University \\ ${ }^{2}$ Xiangya Hospital of Central South University
}

August 27, 2020

\begin{abstract}
Objectives: To summarize the clinical features of patients with hereditary hemorrhagic telangiectasia(HHT) to provide a scientific diagnosis and reduce misdiagnosis for patients with respiratory symptoms of complication of HHT and reduce misdiagnosis. Methods:Retrospective analysis of diagnosis and treatment of two patients with HHT in Xiangya Hospital of Pediatrics and to summarize domestic and international coverage of clinical characteristics of patients with respiratory symptoms of HHT in recent years. Results:patients with HHT have these symptoms: 31 patients Respiratory symptoms of dyspnea (48.43\%), 8 patients shortness (12.50\%), 9 patients chest pain (14.06\%), 4 patients distress (6.25\%), 4 patients chronic cough (6.25\%), 15 patients cyanosis (23.43\%),12 patients hemoptysis (18.75\%), 6 patients of other gastrointestinal symptoms (9.37\%). The main complications were pulmonary hypertension $16(25.00 \%)$ and cardiac dysfunction 12 (18.75\%). The majority of the involved organs were pulmonary arteriovenous malformation (PAVMs) (60.93\%). Computed tomography (CT) scans and contrast echocardiography, CT angiography can be used for detection, which contrast echocardiography is preferred screening methods for PAVMs. The therapy to deal with the symptoms and complications mainly for vascular organ involvement, the principal means for the diagnosis and treatment of mobilization and drug symptomatic treatment, and liver or lung transplant is better. Conclusion The common clinical symptoms of HHT patients with respiratory system involvement are not typical and they are easy to lose early diagnosis. When patients present with respiratory symptoms, the risk of developing severe complications such as pulmonary hypertension and cardiac insufficiency increases. Interventional mobilization is the most effective treatment for PAVMs in HHT patients, but recurrence is possible. Transplantation provides new hope for patients who have failed interventional therapy.
\end{abstract}

\section{Hosted file}

hht.docx available at https://authorea.com/users/354208/articles/477850-hereditaryhemorrhagic-telangiectasia-with-respiratory-symptoms-at-the-first-stage-two-casesreport-and-literature-review 